\title{
Respiratory Rate Monitoring Using Infrared Sensors
}

\author{
Fatih Erden ${ }^{1}$, A. Enis Cetin ${ }^{2}$ \\ ${ }^{1}$ Department of Electrical and Electronics Engineering, Atılım University, Ankara 06836, Turkey \\ erden@ee.bilkent.edu.tr \\ ${ }^{2}$ Department of Electrical and Electronics Engineering, Bilkent University, Ankara 06800, Turkey \\ cetin@bilkent.edu.tr
}

\begin{abstract}
Respiratory rate is an essential parameter in many practical applications such as patient and elderly people monitoring. In this paper, a novel contact-free system is introduced to detect the human breathing activity. The system, which consists of two pyro-electric infrared (PIR) sensors, is capable of estimating the respiratory rate and detecting the sleep apnea. Sensors' signals corresponding to the thoracic movements of a human being are sampled using a microprocessor and analyzed on a general-purpose computer. Sampled signals are processed using empirical mode decomposition (EMD) and a new average magnitude difference function (AMDF) is used to detect the periodicity and the period of the processed signals. The resulting period, by using the fact that breathing is almost a periodic activity, is monitored as the respiratory rate. The new AMDF provides a way to fuse the data from the multiple sensors and generate a more reliable estimation of the respiratory rate.
\end{abstract}

\section{Introduction}

Respiratory rate is an important vital sign in assessing the physical and psychological health of human beings. Respiratory rate, especially the expiratory time is a good predictor of the level of individual anxiety [1]. It is reported in [2] that a serious increase in the breathing activity is the most important indicator of cardiac arrest in hospital wards. It is also known that many lung and heart diseases such as pneumonia affect respiratory rate [3]. Therefore respiratory monitoring is a crucial tool in intensive care units, hospitals, elderly care units, and home medical care services.

Systems for respiratory monitoring can be classified into two categories: (i) systems which are worn on the human's body, e.g. thoracic impedance penumography, photoplethysmography, and (ii) systems which measure the human's near-environment, e.g. sound recording, carbon dioxide sensing. The works in [47] are good examples of the first group. Carmo et al. [4] use a wireless electronic shirt with embedded sensors to measure the heart rate and respiratory frequency. An electromagnetic biosensor centered on the subject's sternum is used for respiratory monitoring in [5]. Peng et al. [6] propose a system with video, pyro-electric infrared (PIR) sensors and wearable actigraphy based monitoring system for sleep monitoring. However it fails to detect the period of breathing and PIR sensors are only used in on/off mode. In [7] a wireless wearable bioimpedance device is developed. But the device basically aims to detect the abnormal breathing waveforms in the bioimpedance signal and cannot estimate the respiratory rate accurately.

The studies in [8-14] exemplify the second group. In [8] and [9], radar sensor based systems for central apnea detection and respiratory monitoring are described, respectively. A biosignal measurement system, which measures the air pressure in an air mattress, to monitor the heartbeat and respiration of a person lying in bed is introduced in [10]. Wang et al. [11] propose a sensor based sleep monitoring system to assess the quantity and quality of sleep for elderly people. But it uses PIR sensors in on/off mode only and does not determine the period of breathing. They also use bed sensors but not for detection of the breathing activity.

Infrared camera based imaging systems are also used to monitor the respiratory activity [12]. Wai et al. [13] describe an array of bed sensors and cameras to monitor client position in bed and different situations when the client is out of bed. While the article describes a bed monitoring system to detect sleep patterns, the system focuses on monitoring falls but not the breathing activity. Furthermore, the use of cameras in bedroom raises issues related to personal privacy of the individual.

In [14], a system consisting of a pyro-electric infrared (PIR) sensor array to monitor the respiratory movements is presented. Fourier analysis of the sensors' signals is used to find out the abnormalities, in particular sleep apnea, in a patient's breathing activity. Respiratory rate measurement is mentioned as a further investigation in the study. In [14], analog signal acquisition from the PIR sensors is not described. In the following section, we present a circuit structure developed for analog data acquisition from a PIR sensor.

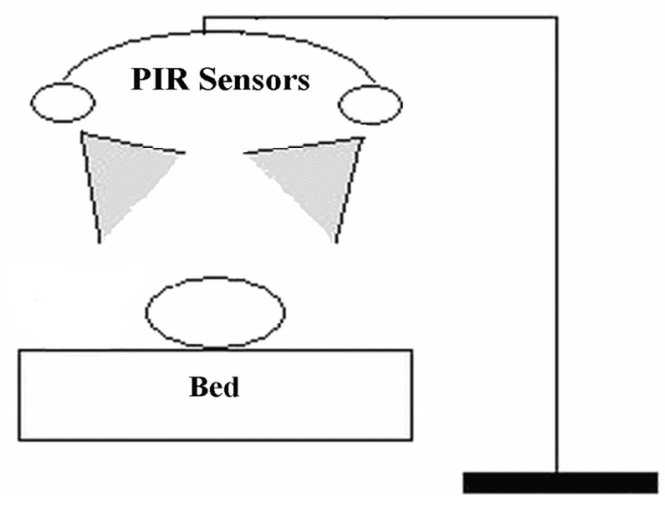

Fig. 1. A typical illustration of the contact-free human breathing activity detection system consisting of two PIR sensors 


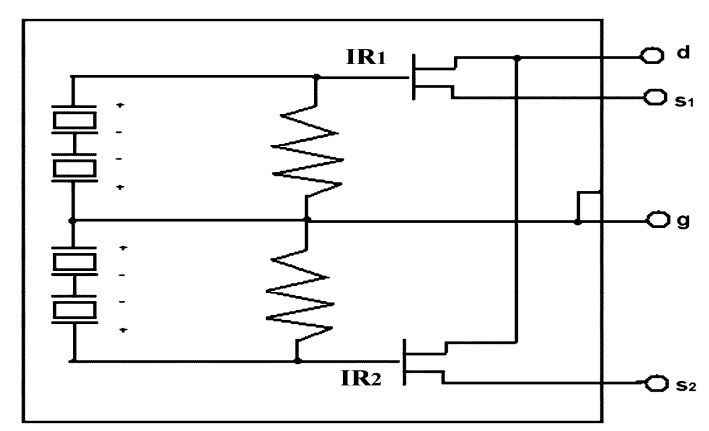

Fig. 2. Model of the inner structure of a differential PIR sensor

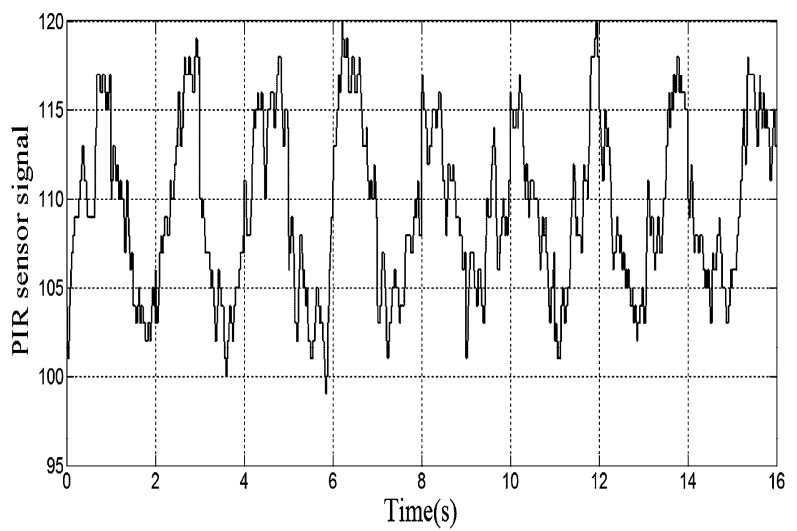

Fig. 3. A typical PIR sensor signal due to a human breathing at a $1 \mathrm{~m}$ distance from the sensor

In this paper, we describe a novel contact-free human breathing activity detection system. The system, which consists of two PIR sensors, is capable of estimating the respiratory rate and detecting the sleep apnea as well. PIR sensors are placed onto a stand near the bed. A typical graphical description of the proposed system setup is shown in Fig. 1. PIR sensors pick up the thoracic movements due to the breathing activity of a human being. Sensor signals are sampled using a microprocessor board and transferred to a general-purpose computer. The sampled signals are processed using empirical mode decomposition (EMD) and fed to a new average magnitude difference function (AMDF) to detect a periodic behavior and the corresponding period. Then, by using the fact that breathing is almost a periodic activity, the period of the processed sensors' signals is monitored as the respiratory rate. The new AMDF provides a way to fuse the data from the multiple sensors and generate a more reliable decision.

The paper is organized as follows: Operating principles of the PIR sensors and data acquisition from the sensors are described in Section 2. EMD based sensor data processing is described in Section 3. Section 4 reports the results of experimental validation and Section 5 concludes the paper.

\section{Data Acquisition}

PIR sensors are widely used commercially for motion detection, e.g. in automation of electrical appliances [15], design and implementation of a home embedded surveillance systems
[16]. These applications are, in general, based on the on/off decisions of the PIR sensors.

A differential PIR sensor basically measures the difference of infrared radiation density between the two pyro-electric elements $\left(\mathrm{IR}_{1}, \mathrm{IR}_{2}\right)$ inside. Fig. 2 shows the block diagram of a typical differential PIR sensor, $\left(\mathrm{s}_{1}\right)$ and $\left(\mathrm{s}_{2}\right)$ are the outputs of the pyro-electric elements and $(\mathrm{g})$ is ground. Normal temperature alterations and changes caused by airflow are cancelled by the two elements connected in parallel. If these elements are exposed to the same amount of infrared radiation, they cancel each other and the sensor produces a zero-output at (d).

Commercially available PIR motion detector circuits produce binary outputs. However, it is possible to capture a continuoustime analog signal representing the amplitude of the voltage signal which corresponds to the transient behavior of the circuit. We use a circuit, which we developed for flickering flame detection [17], to capture an analog output signal from the PIR sensors.

The analog output signal is digitized using a microcontroller with a sampling rate of $20 \mathrm{~Hz}$ and transferred to a generalpurpose computer for further processing. A typical respiratory rate for a healthy adult at rest is $12-20$ breaths per minute. Average resting respiratory rates vary with age. Infants take 30 60 breaths per minute. Therefore even a $2 \mathrm{~Hz}$ sampling frequency is enough for digitization of the analog sensor signal due to Shannon's sampling theorem. However a higher sampling rate $(20 \mathrm{~Hz})$ is selected for digitization. A typical differential PIR sensor signal due to a human breathing at a $1 \mathrm{~m}$ distance from the sensor is shown in Fig. 3. It can be seen from the figure that the signal is almost periodic.

\section{Data Processing}

The human breathing activity is a low-frequency activity with the highest frequency of $1 \mathrm{~Hz}$. Therefore the sampling frequencies of the sensors' output signals should be at least twice that of the highest frequency, as they already are. In addition, the noise is usually a high-frequency activity. Since we are dealing with the almost periodic breathing activity, we reduce the frequency content of the recorded sample signals inside the band of interest using the EMD.

\subsection{Empirical Mode Decomposition}

The EMD method, which is introduced by Huang et al. [18], is used to adaptively decompose a signal into intrinsic oscillations. Because of the simplicity and the efficiency of its algorithm, the EMD is widely used in several fields including biomedical applications $[19,20]$. The EMD decomposes a signal into different frequency bandwidths adaptively.

The EMD method starts by estimating a signal as a sum of a low-frequency and a high-frequency component. The highfrequency component, which is referred to as detail, is obtained by subtracting the low-frequency part of the signal from the original signal. The same procedure is then applied to the lowfrequency part considering it as a new signal to extract a new detail. Given a signal $x(t)$, the EMD algorithm can be summarized as follows [18].

1. Find all maxima and minima of $x(t)$. 
2. Interpolate between minima (maxima) points separately using a cubic spline to obtain the lower (upper) envelope, $e_{\min (\max )}(t)$.

3. Compute the low-frequency component, $m(t)=$ $\left(e_{\min }(t)+e_{\max }(t)\right) / 2$.

4. Extract the detail, $d(t)=x(t)-m(t)$.

5. Iterate on $m(t), x(t)=m(t)$.

Under the zero-mean condition, the detail $d(t)$ is referred to as an Intrinsic Mode Function (IMF). The above procedure is repeated until the IMF definition is met. The output of the EMD of a PIR sensor signal due to a human breathing at a $1 \mathrm{~m}$ distance from the sensor is shown in Fig. 4. The IMF definition is met after 6 iterations of the algorithm, i.e., the dashed line in Fig. 4 is an IMF. Each oscillation of the IMF corresponds to one breathing activity.

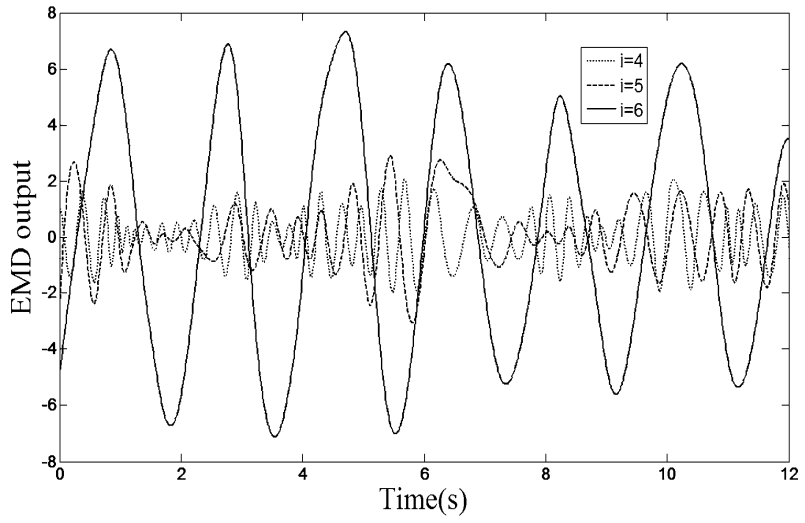

Fig. 4. Output of the EMD of the PIR sensor signal due to a human breathing activity (for 4, 5, and 6 iterations)

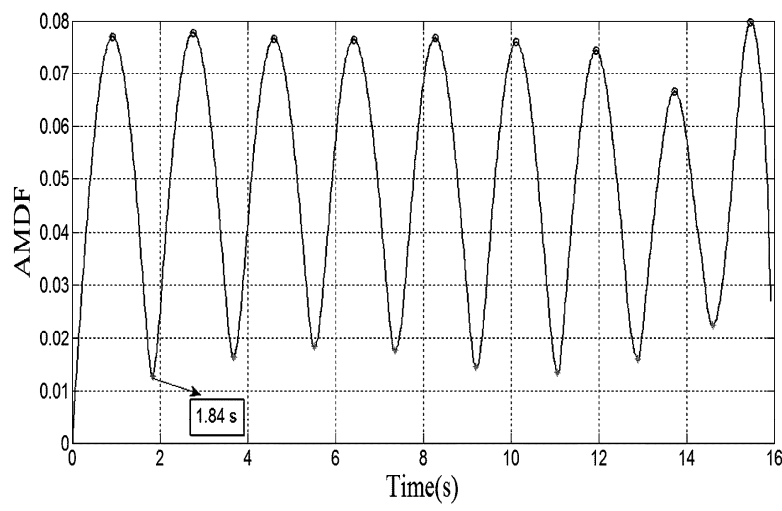

Fig. 5. Output of the AMDF for the IMF in Fig. 4

\subsection{Periodicity Detection Using the AMDF}

Decomposed signals are then fed to the AMDF to detect the periodic behavior. The AMDF is widely used in speech processing for voiced phoneme detection and to determine the period of voiced sounds. The AMDF is defined as follows:

$$
\operatorname{AMDF}[k]=1 / N \sum_{n}|u[n]-u[n-k]|
$$

where $u[n]$ represents the IMF corresponding to one of the PIR sensors and $N$ is the number of samples within the analysis time- window. In this case, the analysis time-window is 1 minute, since we desire to determine the respiratory rate in terms of breathing/minute. Output of the AMDF for the IMF in Fig. 4 is plotted in Fig. 5. AMDF exhibits a set of local minima corresponding to the periods of $u[n]$. We detect the first local minimum and based on the time index of that minimum we determine the period which in turn gives the respiratory rate. For example, the person whose AMDF shown in Fig. 5 takes a breath approximately in every 1.84 seconds. Periodicity can also be detected from the IMF shown in Fig. 4. However, AMDF provides robustness because of data averaging in Eq. 1. Furthermore, it can be computed without performing any multiplications.

We further define a new AMDF function to fuse the data from the two different PIR sensors as follows:

$$
\mathrm{AMDF}_{2}[k]=1 / N \sum_{n} \alpha_{u}|u[n]-u[n-k]|+\alpha_{v}|v[n]-v[n-k]|,
$$

where $u[n]$ is the IMF of one of the PIR sensors, $v[n]$ is the IMF of the other PIR sensor, and $\alpha_{u}$ and $\alpha_{v}$ are some weights. We name the $\mathrm{AMDF}_{2}$ function the multi-modal AMDF because it uses a plurality of sensor signals to determine the periodic or aperiodic nature of the recordings. Obviously, when there is periodicity in either one of the recorded signals, the $\mathrm{AMDF}_{2}$ will exhibit a local minimum. This is important because one of the sensors may not measure a meaningful signal due to the sleeping position of the person. Since the human body has to move somewhat during breathing, one of the PIR sensors will pick up the breathing activity and $\mathrm{AMDF}_{2}$ will produce a local minimum corresponding to the respiratory rate. Another advantage of the $\mathrm{AMDF}_{2}$ is that it can handle phase differences between the sensors' signals which may otherwise result in an inaccurate detection of the respiratory rate.

Table 1. Reliability percentages of respiratory rate

\begin{tabular}{|c|c|c|c|}
\hline Subject Position & $|\epsilon|<0.5$ & $0.5<|\epsilon|<1$ & $1<|\epsilon|<2$ \\
\hline Supine & $68 \%$ & $23 \%$ & $9 \%$ \\
\hline Side-lying & $59 \%$ & $30 \%$ & $11 \%$ \\
\hline
\end{tabular}
measurements for 10 Subjects

\section{Experiments and Results}

Reliability percentages of the respiratory rate measurements for 10 different subjects between ages 10-49 are presented in Table 1. The subjects are asked to lie in supine and side-lying positions, and breathe for one minute. When a person moves during his sleep in the bed, the PIR sensors generate large amplitude signals. Obviously, this time window is not due to the breathing activity and we do not include that portion of the sensor signals to respiratory rate estimation process. The AMDF does not exhibit a local minimum for such windows. During the experiments, the stand holding the PIR sensors is set to be at various distances from $20 \mathrm{~cm}$ to $1 \mathrm{~m}$ from the subject. Experiments include an average of 30 recordings for each subject in each lying-position and more than 600 recordings in total. We observed that none of the estimates differ more than 2 breathings/minute from the ground truth for supine and sidelying positions. In addition, if the accuracy is defined such that the measurement error is less than 1 breathing/minute, the proposed system achieves an average accuracy rate of $90 \%$ in 
the respiratory rate measurements. We also tested the system with people lying in prone position. But since the back movements due to the breathing activity are not too significant to be captured by the PIR sensors, we observed that the system cannot estimate the respiratory rate accurately in prone position.

Each of the PIR sensors is able to estimate the respiratory rate separately. But one of the PIR sensors may fail to detect the breathing activity due to the lying position. The proposed system can handle this problem by using two PIR sensors. As Table 1 reports, the system does not miss any breathing activity due to the sleeping position of a person.

When a person has a sleep apnea, he or she does not move therefore the corresponding IMFs of the PIR sensors' signals become very close to zero. In this case the AMDF function does not have a local minimum in the apnea windows. Its value increases as $k$ increases. One such instance is shown in Fig. 6. As a result the sleep apnea can also be detected by the proposed system.

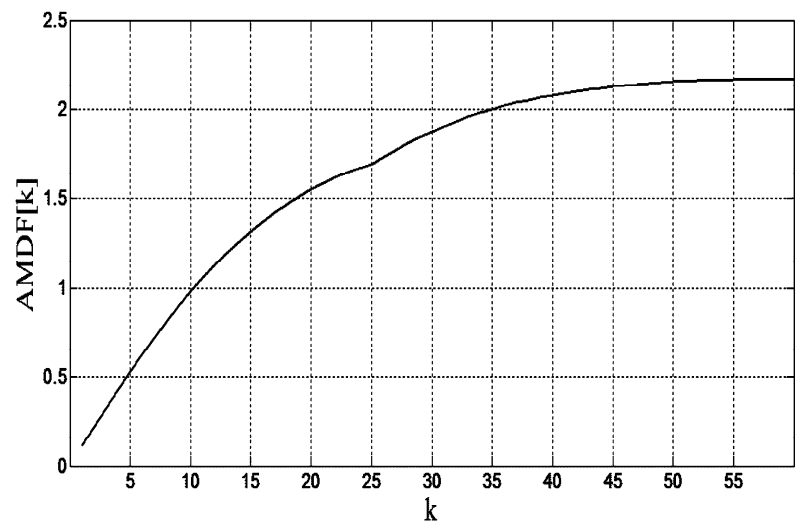

Fig. 6. Output of the AMDF for the IMF of the PIR sensor signal due to a sleep apnea

\section{Conclusions}

In this paper, we proposed a novel system for a reliable contact-free measurement of the respiratory rate. The system consists of two differential PIR sensors. Up to our knowledge, this is the first study that uses the PIR sensors to estimate the respiratory rate. We defined a new multi-modal AMDF function to fuse the data of the multiple sensors. A PIR sensor may not measure a meaningful signal if the thoracic movement due to breathing is not within its field of view. But since the person's body moves noticeably during breathing, one of the PIR sensors picks up the breathing activity and the multi-modal AMDF detects the periodicity and the period of breathing. The proposed system is also able to detect the sleep apnea. The overall system can be implemented using a single low-power microprocessor since both the EMD and the AMDF do not need any complicated data processing operations.

\section{References}

[1] I. Homma, Y. Masaoka, "Breathing rhythms and emotions", Exp. Physiol., vol. 93, no. 9, pp. 1011-1021, Sept. 2008.

[2] M. A. Cretikos, R. Bellomo, K. Hillman, J. Chen, S. Finfer, A. Flabouris, "Respiratory rate: the neglected vital sign", Med. J. Aust., vol. 188, no. 11, pp. 657-659, June 2008.
[3] S. Min, Y. Yun, H. Shin, "Simplified structural textile respiration sensor based on capacitive pressure sensing method", IEEE Sensors J., vol. 14, no. 9, pp. 3245-3251, Sept. 2014.

[4] J. P. Carmo, J. H. Correia, "RF CMOS transceiver at 2.4 $\mathrm{GHz}$ in wearables for measuring the cardio-respiratory function", Measurement, vol. 44, no.1, pp. 65-73, Jan. 2011.

[5] B. Padasdao, E. Shahhaiadar, C. Stickley, O. Boric_Lubecke, "Electromagnetic biosensing of respiratory rate", IEEE Sensors J., vol. 13, no. 11, pp. 4204-4211, Nov. 2013

[6] Y. T. Peng, C. Y. Lin, M. T. Sun, C. A. Landis, "Multimodality sensor system for long-term sleep quality monitoring", IEEE Trans. Bimoed. Circuits Syst., vol. 1, no. 3, pp. 217-227, Sept. 2007.

[7] V. Seppä, J. Väisänen, P. Kauppinen, J. Malmivuo, J. Hyttinen, "Measuring respirational parameters with a wearable bioimpedance device", 13th International Conference on Electrical Bioimpedance and the 8th Conf. Electrical Impedance Tomography, Graz, Astria, 2007, pp. 663-666.

[8] G. P. Weinreich, P. de Chazal, Y. Wang, S. Teschler, H. Teschler, "A pilot study on non-contacting detection of central apneas", World Congress on Medical Physics and Biomedical Engineering, Munich, Germany, 2009, pp. 790791.

[9] D. Zito, D. Pepe, M. Mincica, F. Zito, A. Tognetti, A. Lanata, D. De Rossi, "SoC CMOS UWB Pulse radar sensor for contactless respiratory rate monitoring", IEEE Trans. Biomed. Circuits Syst., vol. 55, no. 6, pp. 503-510, Dec. 2011.

[10] Y. Kurihara, K. Watanabe, "Development of unconstrained heartbeat and respiration measurement system with pneumatic flow", IEEE Trans. Biomed. Circuits Syst., vol. 6, no. 6, pp. 503-510, Dec. 2012.

[11] H. Wang, H. Zheng, J. C. Augusto, S. Martin, M. Mulvenna, W. Carswell, J. Wallace, P. Jeffers, B. Taylor, $\mathrm{K}$. McSorley, "Monitoring and analysis of sleep pattern for people with early dementia", IEEE International Conference on Bioinformatics and Biomedicine Workshops, Hong Kong, 2010, pp. 405-410.

[12] J. N. Murthy, J. van Jaarsveld, J. Fei, I. Pavlidis, R. I. Harrykissoon, J. F. Lucke, S. Faiz, R. J. Castriotta, "Thermal infrared imaging: a novel method to monitor airflow during polysomnography", Sleep, vol. 32, no. 11, pp. 1521-1527, Nov. 2009.

[13] A. A. P. Wai, W. Huang, V. F. S. Fook, J. Biswas, H. ChiChun, L. Koujuch, "Situation-aware patient monitoring in and around the bed using multimodal sensing intelligence", IEEE International Conference on Intelligent Environments, Kuala Lumpur, Malaysia, 2010, pp. 128 133.

[14] V. Hers, D. Corbugy, I. Joslet, P. Hermant, J. Demerteau, B. Delhougne, G. Vandermoten, J. P. Hermanne, "New concept using Passive Infrared (PIR) technology for a contactless detection of breathing movement: A pilot study involving a cohort of 169 adult patients", J. Clin. Monit. Comput., vol. 27, no. 5, pp. 521-529, Oct. 2013.

[15] R. Samson, T. Winings, "Automated dispenser for disinfectant with proximity sensor," U.S. Patent 5695 091, Dec. 9, 1997. 
[16] Y. W. Bai, L. S. Shen, Z. H. Li, "Design and implementation of an embedded home surveillance system by use of multiple ultrasonic sensors", IEEE Trans. Consum. Electron., vol. 56, no. 1, pp. 119-124, Feb. 2010.

[17] F. Erden, B. U. Toreyin, E. B. Soyer, I. Inac, O. Gunay, K. Kose, A. E. Cetin, "Wavelet based flickering flame detector using differential PIR sensors", Fire Saf. J., vol. 53, pp. 1318, Oct. 2012.

[18] N. E. Huang, Z. Shen, S. R. Long, M. C. Wu, H. H. Shih, Q. Zheng, N-C. Yen, C. C. Tung, H. H. Liu, "The empirical mode decomposition and Hilbert spectrum for non-linear and non-stationary time series analysis", Royal Society of London A: Mathematical, Physical and Engineering Sciences, 1998, pp. 903-995.

[19] C. D. Papadaniil, L. J. Hadjileontiadis, "Efficient heart sound segmentation and extraction using ensemble empirical mode decomposition and Kurtosis features", IEEE J. Biomed. Health Inform., vol. 18, no. 4, pp. 11381152, July 2014

[20] K. T. Sweeney, S. F. McLoone, T. E. Ward, "The use of ensemble empirical mode decomposition with canonical correlation analysis as a novel artifact removal technique", IEEE Trans. Biomed. Eng., vol. 60, no. 1, pp. 97-105, Jan. 2013 